\title{
Elevated fibrinogen-like protein 2 in TNBS-induced colitis mice: Association with Th17 and regulatory T cells
}

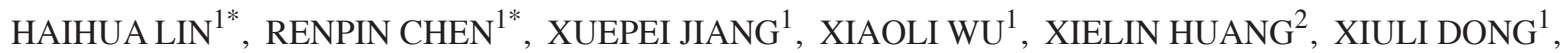

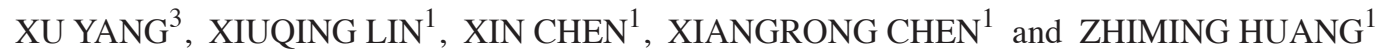 \\ ${ }^{1}$ Department of Gastroenterology, The First Affiliated Hospital of Wenzhou Medical University; \\ ${ }^{2}$ Department of Clinical Medicine, Renji College of Wenzhou Medical University; ${ }^{3}$ Center of Reproductive Medicine, \\ The First Affiliated Hospital of Wenzhou Medical University, Wenzhou, Zhejiang 325035, P.R. China
}

Received August 13,2016; Accepted May 11, 2017

DOI: $10.3892 / \mathrm{mmr} .2017 .7005$

\begin{abstract}
The etiology and pathogenesis of inflammatory bowel disease (IBD) is complex and remains to be completely elucidated. Numerous cytokines are associated with the initiation and development of IBD. Fibrinogen-like protein 2 (FGL2), an immunosuppressive cytokine expressed by regulatory cluster of differentiation (CD) $4^{+} \mathrm{T}$ (Treg) cells, has been identified to be important for immunomodulatory activity in the inflammatory state. Therefore, the present study investigated the expression of FGL2 and interleukin (IL)-17 in trinitro-benzene-sulfonic acid (TNBS)-induced colitis mice to identify the function of FGL2, based on the effector CD4 ${ }^{+}$T helper (Th)17/Treg balance, in IBD. Compared with control mice, TNBS-induced mice exhibited marked alterations in clinical manifestation, including macroscopic and histopathological damage. Intestinal and peripheral expression of FGL2 was upregulated in TNBS-induced mice, while the level of FGL2 in the spleen was decreased. Expression of IL-17 in the plasma, colon and spleen was increased in TNBS-induced mice. The percentage of Treg cells was markedly decreased, although Th17 cells were increased following TNBS induction. The results of the present
\end{abstract}

Correspondence to: Professor Zhiming Huang or Professor Xiangrong Chen, Department of Gastroenterology, The First Affiliated Hospital of Wenzhou Medical University, South Baixiang Street, Shangcai, Ouhai, Wenzhou, Zhejiang 325035, P.R. China

E-mail: wzhospitalhzm@126.com

E-mail: doctorcxr@126.com

${ }^{*}$ Contributed equally

Abbreviations: IBD, inflammatory bowel disease; FGL2, fibrinogen-like protein 2; Treg, regulatory cluster of differentiation $4^{+}$ T cells; IL-17, interleukin17; Th17, effector cluster of differentiation $4^{+} \mathrm{T}$ helper 17; TNBS, trinitro-benzene-sulfonic acid; TNF- $\alpha$, tumor necrosis factor- $\alpha$; RT, room temperature; PMA, phorbol 12-myristate 13 -acetate; ROR- $\gamma \mathrm{t}$, retinoic acid related orphan receptor- $\gamma \mathrm{t}$; Foxp3, forkhead box protein 3 ; TGF- $\beta$, transforming growth factor- $\beta$

Key words: FGL2, IL-17, IBD, regulatory T cells, Th17 effector cells study indicated that, in the colitis model, FGL2 was associated with the immunopathogenesis of IBD.

\section{Introduction}

Inflammatory bowel disease (IBD) is the term for chronic and relapsing-remitting inflammatory disorders of the gastrointestinal tract, including Crohn's disease and ulcerative colitis $(1,2)$. While the incidence and prevalence of IBD are increasing, the etiology and pathogenesis are complex and remain unclear $(3,4)$. Various factors, including immunity, heredity, the intestinal environment (defective epithelial barrier and increased intestinal permeability) and bacterial infection, appear to be associated with the pathogenesis of IBD, particularly immunological factors $(1,5)$.

The hypothesis that dysfunction of the immune system and disproportionality of pro- and anti-inflammatory mediators serve important roles in the pathogenesis of IBD has been widely accepted $(6,7)$. Naïve cluster of differentiation (CD) $4^{+} \mathrm{T}$ cells generally divide into different lineages, which encompass effector $\mathrm{CD}^{+}{ }^{+} \mathrm{T}$ helper $(\mathrm{Th})$ cells and regulatory $\mathrm{CD}^{+} \mathrm{T}$ (Treg) cells, and vary in cytokine production and function $(8,9)$. Apart from classical Th1 and Th2 cells, Th17 lymphocytes are a novel subpopulation of effector Th cells that have been demonstrated to be an important pathogenic element in IBD (5). In addition, Th17 cells are characterized by the production of a large amount of interleukin (IL)-17A, IL-17F, IL-21, IL-22 and IL-23, and have been observed in the mucosa and lamina propria of patients with IBD (1,10-12). By contrast, Treg cells produce a number of anti-inflammatory cytokines which suppress the activation and effector functions of $\mathrm{T}$ cells. It has been reported that Treg cells inhibit abnormal inflammatory responses against the commensal flora or dietary antigens, and are involved in the maintenance of intestinal homeostasis $(1,13)$. Therefore, the quantity and function of Treg cells may be associated with the initiation and progression of IBD (14). A previous study demonstrated that breakdown of the Th17/Treg balance may promote inflammation and autoimmune disease, including IBD (15).

IL-17, an important pro-inflammatory cytokine secreted by Th17, is associated with a number of chronic inflammatory disorders $(15,16)$. It has been observed that IL-17 is 
overexpressed in patients with IBD (1). Additionally, fibrinogen-like protein 2 (FGL2) is one of the immunosuppressive cytokines expressed by Treg cells and has been identified to be an important effector molecule by which Treg cells exerts their immunosuppressive effects (17-20). FGL2 has been demonstrated to inhibit the proliferation of $\mathrm{T}$ cells and the maturation of bone marrow-derived cells. Previous studies have demonstrated that subsets of Treg cells with increased levels of FGL2 are more suppressive and that Treg cells from FGL $^{-1-}$ mice have been observed exhibit decreased suppressive activity $(17,20,21)$. It was previously identified that the expression of FGL2 in intestinal mucosal biopsies and peripheral blood was increased in patients with active disease, and decreased in inactive disease. Additionally, FGL2 levels were significantly positively correlated with clinical disease activity index, erythrocyte sedimentation rate and C-reactive protein levels (22). Therefore, it was hypothesized that the increased levels of FGL2 may be associated with a mass of Treg cells recruited to regions of inflammation to counter inappropriately activated effector T cells. In addition, Dothel et al (2) demonstrated that acute inflammation in animal models of trinitro-benzene-sulfonic acid (TNBS)-induced colitis is characterized by a Th1/Th17-oriented response, with increased levels of tumor necrosis factor (TNF)- $\alpha$, IL-1 $\beta$, IL-12, IL-17, IL-18 and IL-6. It has been additionally suggested that Crohn's disease is oriented to a Th1 and Th17 immune response and, in order to mimic the chronic inflammation typical of Crohn's disease, models have been developed via multiple TNBS administrations. Therefore, the TNBS-induced model presented in the present study is a model of the acute phase of Crohn's disease $(2,23)$.

The present study investigated alterations in FGL2 and IL-17 expression in the TNBS-induced colitis model, in order to identify whether the balance between Th17 and Treg was disrupted and whether the imbalance may be involved in the immunopathogenesis of IBD. Therefore, the purpose of the present study was to demonstrate variation in FGL2 and IL-17 expression in an animal model of IBD and to examine their involvement in the immunopathogenesis of IBD.

\section{Materials and methods}

Animals. A total of $22 \mathrm{BALB} / \mathrm{c}$ mice of $6-8$ weeks of age (male, $\sim 20 \mathrm{~g}$ ) were purchased from Shanghai SLAC Laboratory Animal Co., Ltd [Shanghai, China; license no. SCXK (Shanghai) 2012-0002]. Animals were housed in standard cages (5 mice/cage) under specific pathogen-free conditions in the Laboratory Animal Research Center of Wenzhou Medical University (Wenzhou, China). Mice were given free access to autoclaved tap water and to a standard diet, maintained under controlled conditions of light (12-h light/dark cycle), temperature $\left(22-24^{\circ} \mathrm{C}\right)$ and humidity (45-55\%). All procedures were conducted ethically according to the Guide for the Care and Use of the Administration Committee of Experimental Animals of Wenzhou Medical University (permit no. wydw2015-0123).

TNBS-induced colitis. Mice were skin sensitized with a mixture of acetone, olive oil and TNBS (Sigma-Aldrich; Merck KGaA, Darmstadt, Germany; 64:16:20 v/v/v), prior to
Table I. Primers used for the reverse transcription-quantitative polymerase chain reaction.

Gene Primer sequence

GAPDH sense: 5'-GGTTGTCTCCTGCGACTTCA-3 antisense:5'-TGGTCCAGGGTTTCTTACTCC-3'

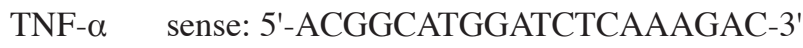
antisense:5'-GTGGGTGAGGAGCACGTAGT-3'

ROR- $\gamma \mathrm{t}$ sense: 5'-GAACCAGAACAGGGTCCAGA-3' antisense:5'-TCGGAAGGACTTGCAGACAT-3'

Foxp3 sense: 5'-ACTCGCATGTTCGCCTACTT-3' antisense:5'-GTCCACACTGCTCCCTTCTC-3'

FGL2 sense: 5'-ATTAGATGTTGAACTGGCTGTGA-3' antisense:5'-TGGCAAATCTAACCGTTGTGG-3'

IL-17 sense: 5'-TCCCTCTGTGATCTGGGAAG-3' antisense:5'-CTCGACCCTGAAAGTGAAGG-3'

TNF- $\alpha$, tumor necrosis factor- $\alpha$; ROR- $\gamma$ t, retinoic acid related orphan receptor- $\gamma \mathrm{t}$; Foxp3, forkhead box protein 3; FGL2, fibrinogen-like protein 2; IL-17, interleukin-17.

TNBS administration. A total of 7 days subsequently, mice were weighed and anesthetized by intraperitoneal injection of ketamine/xylazine solution ( $80 \mathrm{ml} / 10 \mathrm{~g}$ body weight). TNBS was dissolved in anhydrous ethanol $(50: 50 \mathrm{v} / \mathrm{v})$. A total of $100 \mu \mathrm{l}$ TNBS solution $(100 \mathrm{mg} / \mathrm{kg}$ body weight) was administered intrarectally (via $3.5 \mathrm{~F}$ catheter) to mice maintained for $60 \mathrm{sec}$ in a vertical position. The catheter was inserted into the colon $4 \mathrm{~cm}$ proximal to the anus. Control mice received $100 \mu \mathrm{l}$ $0.9 \%$ saline intrarectally (24). The sample size in each group was 8 .

Clinical and macroscopic analysis of colitis. The clinical analysis of colitis was performed by daily monitoring of body weight, diarrhea and hemafecia (25). Loss of body weight was calculated as the percentage difference relative to initial body weight. Diarrhea was scored as follows: 0, Normal; 2, loose stools; and 4, diarrhea that remained adhesive to the anus. Bleeding was scored as follows: 0 , Negative hemoccult test; 2 , positive hemoccult test; and 4, apparent bleeding (23). Mice were euthanized by cervical dislocation 3 days subsequent to TNBS administration, when the inflammation was most severe. The colon was removed and opened longitudinally. The macroscopic damage was assessed by a blinded observer with the following score system $(5,26)$ : 0 , Normal; 1 , hyperemia, edema, no ulcer; 2 , hyperemia, edema, small linear ulcers or petechiae; 3 , hyperemia, edema, wide ulcers, necrosis or adhesions; and 4, hyperemia, edema, megacolon, stenosis or perforation.

Histology. For histological analysis, the colonic fragments $(0.5 \mathrm{~cm})$ were fixed in $4 \%$ paraformaldehyde at $4^{\circ} \mathrm{C}$ for $48 \mathrm{~h}$, dehydrated, embedded in paraffin, sectioned (4- $\mu \mathrm{m}$ thickness), and stained with hematoxylin and eosin. The pathological slices were observed under a light microscope at a magnification of $\mathrm{x} 200$ by two blinded pathologists and the microscopic damage was scored as follows (27): 0 , No evidence of 
A

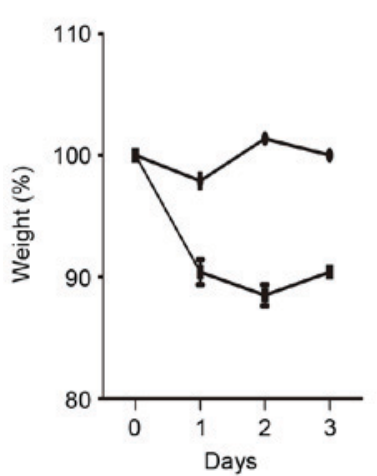

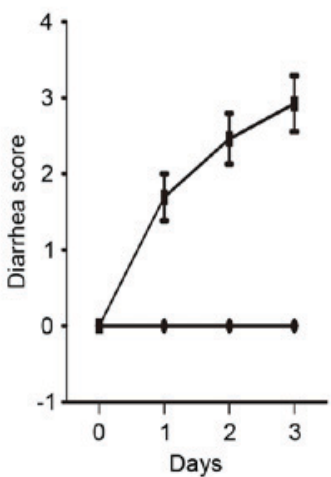

c

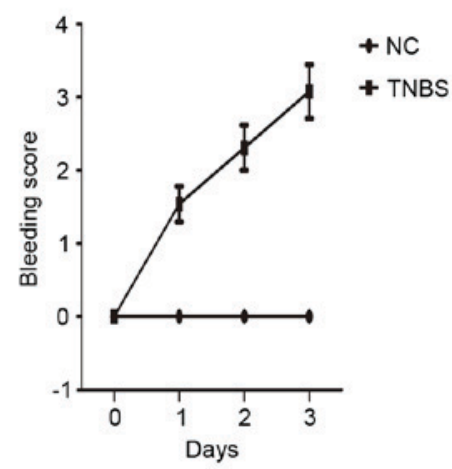

D

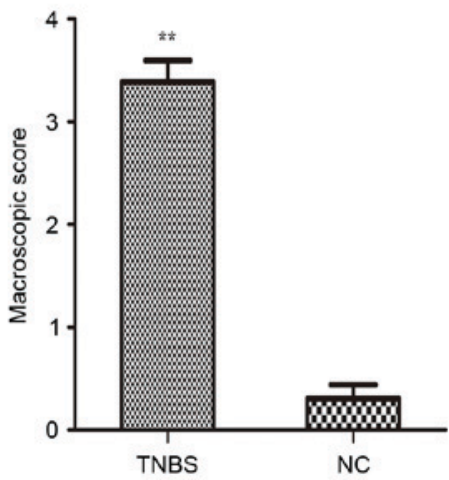

Figure 1. Clinical characteristics of TNBS-induced inflammatory bowel disease model mice. Mice were treated with TNBS (100 mg/kg), and observed and weighed every day until euthanized. (A) Body weight alterations (percentage of original body weight); (B) bleeding score; (C) diarrhea score; and (D) representative macroscopic images of different colons at 3 days post-TNBS administration. The macroscopic damage was scored. ${ }^{* *} \mathrm{P}<0.001 \mathrm{vs}$. NC. $\mathrm{n}=8 \mathrm{mice} / \mathrm{group}$. TNBS, trinitro-benzene-sulfonic acid; NC, normal control.

inflammation; 1, low level of inflammation with scattered infiltrating mononuclear cells (1-2 foci); 2, moderate inflammation with multiple foci; 3 , high level of inflammation with increased vascular density and marked wall thickening; and 4, maximal severity of inflammation with transmural leukocyte infiltration and loss of goblet cells.

Immunohistochemistry. The colon specimens were fixed with $4 \%$ paraformaldehyde at $4^{\circ} \mathrm{C}$ for $48 \mathrm{~h}$, embedded with paraffin, and sectioned (4- $\mu \mathrm{m}$ thickness) for immunohistochemical staining of FGL2, IL-17 and TNF- $\alpha$. Following incubation with xylene and descending concentrations of ethanol, antigens were retrieved using citrate buffer for $15 \mathrm{~min}$ at $100^{\circ} \mathrm{C}$. Endogenous peroxidases were removed in $3 \%$ hydrogen peroxidase for $15 \mathrm{~min}$ at room temperature (RT) followed by $5 \%$ goat serum (Beijing Solarbio Science $\&$ Technology Co., Ltd., Beijing, China) for $1 \mathrm{~h}$ at $37^{\circ} \mathrm{C}$ for blocking. Sections were incubated with antibodies [FGL2 (cat. no. ab198029; 1:100), TNF- $\alpha$ (cat. no. ab6671; 1:250) and IL-17 (cat. no. ab79056; 1:300) (all Abcam, Cambridge, UK)] overnight at $4^{\circ} \mathrm{C}$, and subsequently incubated with horseradish peroxidase-conjugated secondary antibody (PV.6001; 1:100; Zhongshan Golden Bridge Biotechnology, Beijing, China) for $30 \mathrm{~min}$ at $37^{\circ} \mathrm{C}$. Antibody bindings were counterstained with $10 \%$ hematoxylin at room temperature for $2 \mathrm{sec}$, dehydrated with ascending concentrations of ethanol, cleared with xylene and mounted. A negative control was performed according to the same procedure. Images were captured using a biological imaging microscope at a magnification of $\mathrm{x} 200$ or $\mathrm{x} 400$ (BX53; Olympus Corporation, Tokyo, Japan).

Western blot analysis. Total proteins of colon specimens were extracted in radioimmunoprecipitation lysis buffer (Beijing Solarbio Science \& Technology Co., Ltd.) and phenylmethylsulfonyl fluoride. The protein concentration was analyzed by a BCA kit (Tiangen Biotechnology, Beijing, China). Equal amounts of proteins $(40 \mu \mathrm{g})$ were separated using SDS-PAGE on a $12 \%$ gel and transferred onto polyvinylidene fluoride membrane (EMD Millipore, Billerica, MA, USA). Following blocking in 5\% non-fat milk for $90 \mathrm{~min}$ at room temperature, the membranes were incubated overnight at $4^{\circ} \mathrm{C}$ with anti-FGL2 (polyclonal; rabbit anti-mouse; ab198029; 1:1,000), anti-TNF- $\alpha$ (polyclonal; rabbit anti-mouse; ab667; 1:1,000), anti-IL-17 (polyclonal; rabbit anti-mouse; ab79056; 1:1,000) (all Abcam) and anti-GAPDH (polyclonal; rabbit anti-mouse; BS60630; 1:5,000; Bioworld Technology, Inc., St. Louis Park, MN, USA), and washed three times in TBS with Tween-20. The membranes were subsequently incubated with secondary antibodies conjugated to horseradish peroxidase (7074; 1:5,000; Cell Signaling Technology, Inc., Danvers, MA, USA) for $1 \mathrm{~h}$ at RT, followed by washing three times. Immunoreactive bands were visualized using an enhanced chemiluminescence kit (Bio-Rad Laboratories, Inc., Hercules, CA, USA). Image Lab software version 4.1 (Bio-Rad Laboratories, Inc.) was used for densitometric analysis. 
A

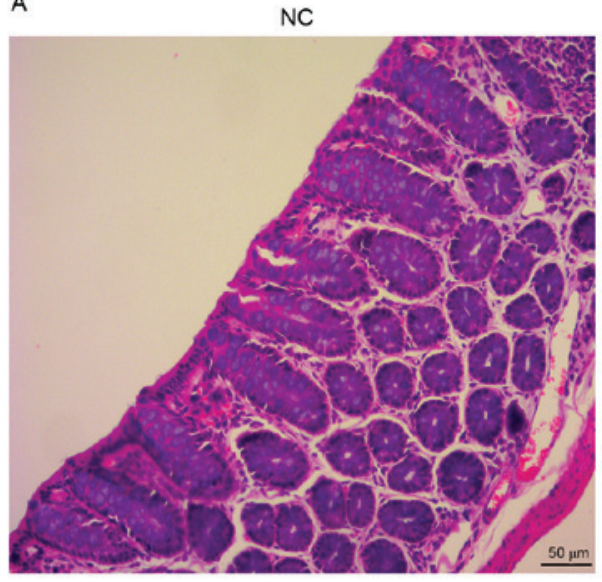

C
B TNBS

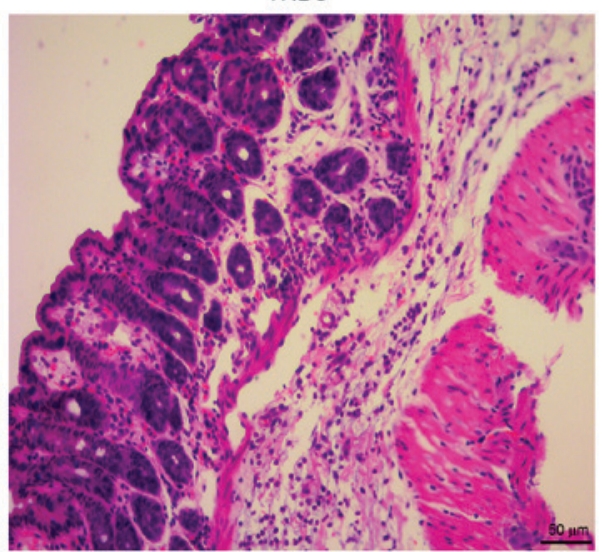

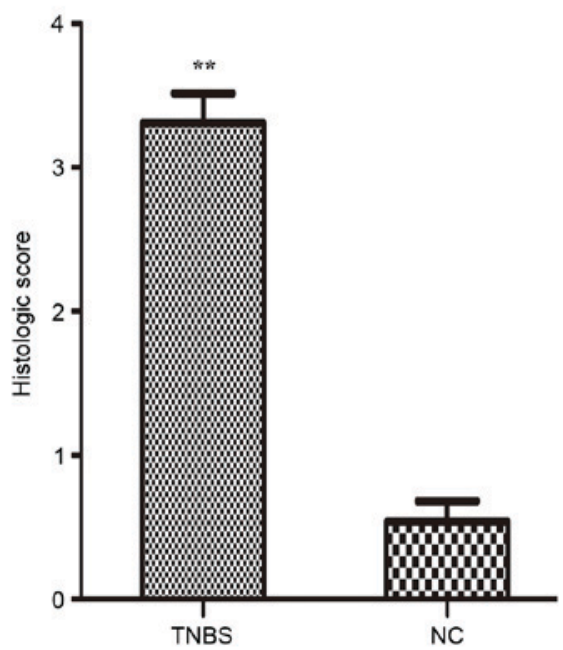

Figure 2. Histological analysis. Representative hematoxylin and eosin-stained colon sections are presented (original magnification, x200). (A) Colon sections from the control group. (B) Colon sections from the TNBS-induced group. (C) Histological scores for colons. ** P<0.001 vs. NC. $n=8$ mice/group. TNBS, trinitro-benzene-sulfonic acid; $\mathrm{NC}$, normal control.

Reverse transcription-quantitative polymerase chain reaction $(R T-q P C R)$. Total RNA was extracted from the colon tissue and spleen mononuclear cells using TRIzol reagent (Invitrogen; Thermo Fisher Scientific, Inc., Waltham, MA, USA), according to the manufacturer's protocol. A total of $1 \mu \mathrm{g}$ total RNA was reverse-transcribed into cDNA using the RevertAid First Strand cDNA Synthesis kit (K1622; Thermo Fisher Scientific, Inc.). The mRNA expression of a number of genes was quantified using SYBR Green Real-time PCR Master Mix Plus (Applied Biosystems; Thermo Fisher Scientific, Inc.) on an ABI 7500 Sequence-Detection System (Applied Biosystems; Thermo Fisher Scientific, Inc.). The PCR cycling conditions were as follows: An initial denaturation and activation at $95^{\circ} \mathrm{C}$ for $10 \mathrm{~min}$, followed by 40 amplification cycles of $95^{\circ} \mathrm{C}$ for $15 \mathrm{sec}$ and $60^{\circ} \mathrm{C}$ for $60 \mathrm{sec}$. The primer sequences are listed in Table I. GAPDH was used as reference gene. Relative gene expression levels were calculated using the $2^{-\Delta \Delta \mathrm{Cq}}$ method (28).

ELISA analysis. Peripheral blood from the mice was drawn into EDTA-anticoagulant tubes, and centrifuged for $15 \mathrm{~min}$ $(3,000 \mathrm{x} \mathrm{g})$ at $4^{\circ} \mathrm{C}$. The plasma was collected and stored at $-80^{\circ} \mathrm{C}$ until tested. ELISA kits were used to assess the plasma concentrations of FGL2 (BPE10791R; Shanghai Boyun Biotech Co., Ltd., Shanghai China), IL-17 (BPE10871R; Shanghai Boyun Biotechnology Co., Ltd.) and TNF- $\alpha$ (BPE10912R; Shanghai Boyun Biotechnology Co., Ltd.), according to the manufacturer's protocol.

Flow cytometric analysis. The spleen was removed from mice 3 days subsequent to TNBS administration and prepared into spleen mononuclear cells The spleen was milled into a homogenate and gradiently centrifuged for $20 \mathrm{~min}(716 \mathrm{x} \mathrm{g})$ at room temperature with lymphocyte separation solution (Tianjin Haoyang Biotechnology Co., Ltd., Tianjin China) leaving the spleen mononuclear cells in the middle of the centrifugal layer. For analysis of Th17 cells, cells were stimulated with phorbol 12-myristate 13-acetate (PMA; $50 \mathrm{ng} / \mathrm{ml}$ ) and ionomycin $(1 \mu \mathrm{g} / \mathrm{ml})$ in the presence of brefeldin A $(10 \mu \mathrm{g} / \mathrm{ml})$ and monensin $(1.4 \mu \mathrm{g} / \mathrm{ml})$, at $37^{\circ} \mathrm{C}$ with $5 \% \mathrm{CO}_{2}$ for $4 \mathrm{~h}$. Cells were washed with PBS and surface-labeled with fluorescein isothiocyanate-(FITC-) conjugated anti-CD4 (1:200; 85-11-0041-81; eBioscience, San Diego, CA, USA) for $40 \mathrm{~min}$ at $4^{\circ} \mathrm{C}$. Following fixing and permeabilizing using a fixation/permeabilization buffer (BD Biosciences, San Jose, CA, USA), cells were labeled with phycoerythrin-(PE-) 

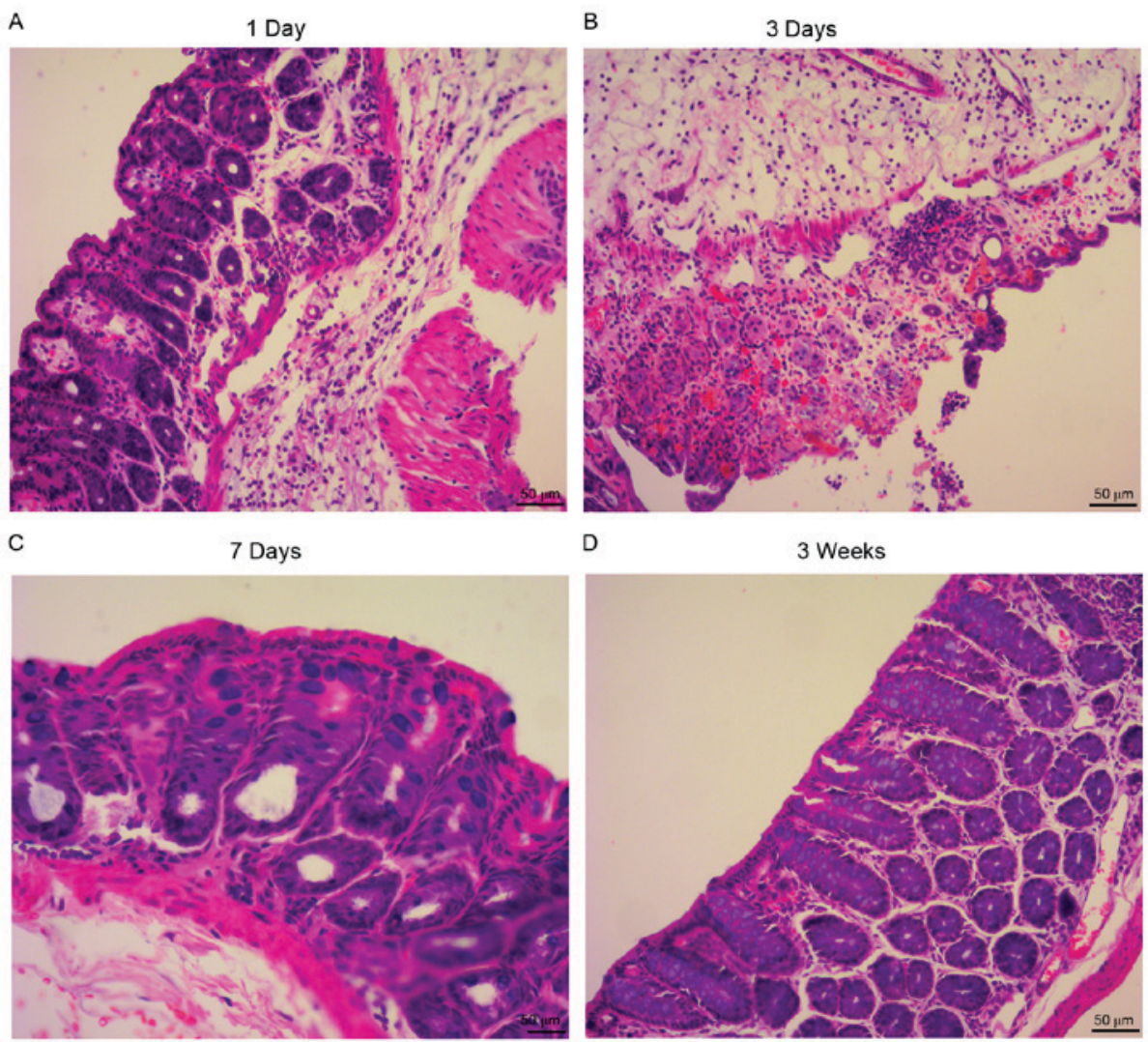

Figure 3. Analysis of microscopic damage to the colons of TNBS-treated mice. Representative hematoxylin and eosin-stained colon sections at different time points are presented. The decrease in goblet cells, loss of crypts, damage to crypts, infiltration by inflammatory cells and destruction of the mucosal layer was observed at (A) day 1 post-TNBS administration, and was observed to be more severe at (B) day 3 . The inflammatory damage was observed to be repaired gradually at (C) day 7 and at (D) 3 weeks post-TNBS administration. Scale bar, $50 \mu \mathrm{m}$. TNBS, trinitro-benzene-sulfonic acid.

conjugated anti-IL-17 (1:100; 85-12-7177-81; eBioscience) for $40 \mathrm{~min}$ at $4^{\circ} \mathrm{C}$. For analysis of Treg cells, without PMA and ionomycin stimulation, surface staining was performed with FITC-conjugated anti-CD4 (1:200; 85-11-0041-81; eBioscience) and allophycocyanin-conjugated anti-CD25 (1:67; 85-17-0390-82; eBioscience) for $40 \mathrm{~min}$ at $4^{\circ} \mathrm{C}$. Subsequently, cells were fixed and permeabilized, and intracellular staining was performed with PE-conjugated anti-forkhead box protein 3 (Foxp3; 1:80; 85-12-5773-82; eBioscience) for $40 \mathrm{~min}$ at $4^{\circ} \mathrm{C}$. Appropriate isotype controls were used in the experiments. The stained cells were assessed using a FACSCalibur flow cytometer (BD Biosciences) and the data were analyzed using FlowJo software (version 7.6.1; Tree Star, Inc., Ashland, OR, USA).

Statistical analysis. Statistical analysis was performed using SPSS software version 16.0 (SPSS Inc., Chicago, IL, USA). Data were analyzed using the Student's t-test for two-group comparisons. Results are presented as the mean \pm standard error of the mean and $\mathrm{P}<0.05$ was considered to indicate a statistically significant difference.

Ethical considerations. In accordance with the Association for the Assessment and Accreditation of Laboratory Animal Care International (Frederick, MD, USA), mice were maintained under specific pathogen-free conditions and studied according to protocols approved by the Wenzhou Medical University Animal Care and Use Committee.

\section{Results}

TNBS-induced mice develop severe clinical manifestations and gross morphological alterations. The clinical symptoms and macroscopic scores in TNBS-induced colitis were evaluated. The mice exhibited a disease phenotype characterized by body weight loss, diarrhea, defecate occult blood or hemafecia following TNBS administration (Fig. 1A-C). In addition, $40 \%$ mortality was observed relative to the saline control $(\mathrm{P}<0.05)$. Body weight loss began immediately in the TNBS-induced mice. By contrast, the control group did not develop colitis or succumb to the treatment. Subsequently, the colonic samples were observed and scored by a blinded observer (Fig. 1D).

Histological alterations and inflammation score. In accordance with the macroscopic test, it was observed that the colon of the IBD model animals exhibited hyperemia, edema, ulcers, necrosis and adhesion. Histological examination demonstrated alterations in the TNBS-induced mice, including a decrease in goblet cells, loss of crypts, damage to crypts, infiltration by inflammatory cells and extensive destruction of the mucosal layer (Fig. 2A and B). Histological scoring was performed as described above. The results of the present study demonstrated that the histological scores of the TNBS-induced mice were markedly increased, compared with the control group (Fig. 2C). The inflammation was most severe 3 days subsequent to TNBS administration, compared with other time points (days 1 and 7, and 3 weeks; Fig. 3). 
A

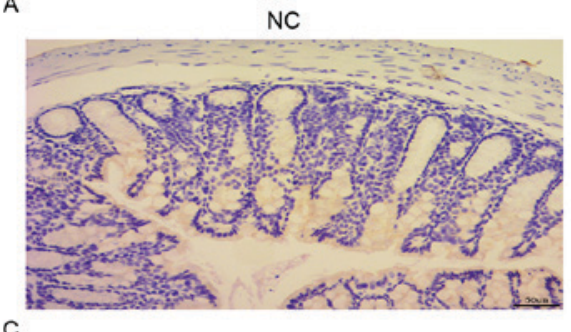

c

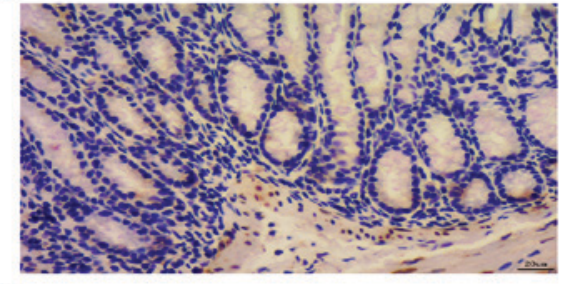

E

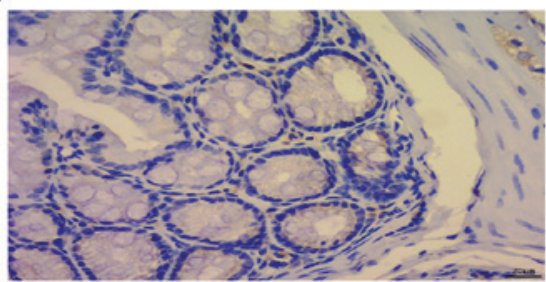

B

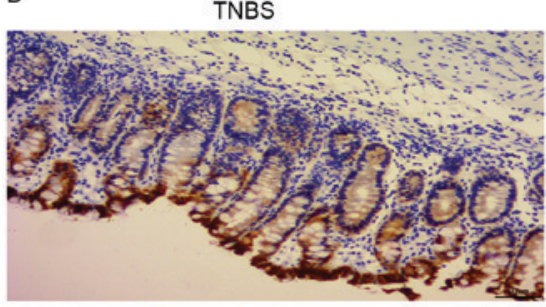

D

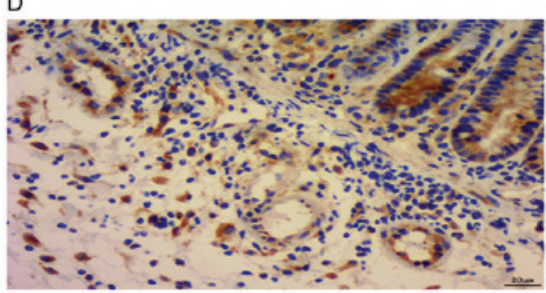

F

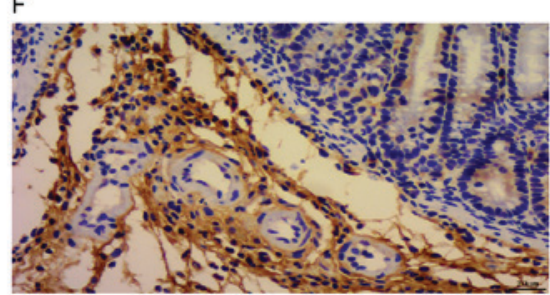

G

NC
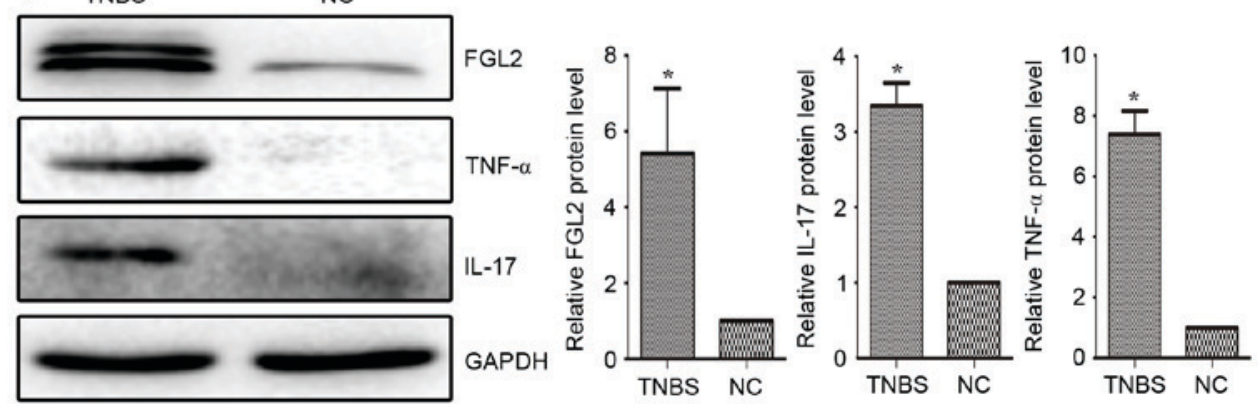

Figure 4. Immunohistochemical analysis. Immunohistochemical staining of colon slices for FGL2 in the (A) NC and (B) TNBS groups (magnification, x200); IL-17 in the (C) NC and (D) TNBS groups (magnification, x400); and TNF- $\alpha$ in the (E) NC and (F) TNBS groups (magnification, $x 400$ ). The NC group exhibited little or no positive expression. The TNBS-induced exhibited marked expression. (G) Western blot analysis of the protein expression of FGL2, IL-17 and TNF- $\alpha$ in the colon was performed and quantified. ${ }^{*} \mathrm{P}<0.05$ vs. NC. $\mathrm{n}=8$ mice/group. TNBS, trinitro-benzene-sulfonic acid; NC, normal control; TNF- $\alpha$, tumor necrosis factor- $\alpha$; FGL2, fibrinogen-like protein 2; IL-17, interleukin-17.

FGL2 expression is increased in the colon tissue from TNBS-induced mice, accompanied by elevated pro-inflammatory cytokine expression. Compared with the control group, pro-inflammatory cytokine expression in the colon tissue, including IL-17 and TNF- $\alpha$, was significantly increased on day 3 following TNBS administration. The expression of FGL2 exhibited the same trend as IL-17 and TNF- $\alpha$, which was consistent with a previous study in patients with IBD (22). The results of the immunohistochemistry and western blotting of FGL2, IL-17 and TNF- $\alpha$ in the present study demonstrated upregulated expression in colonic sections from TNBS-induced colitis mice, compared with the control group (Fig. 4).

MRNA levels of Th17 and Treg transcription factors and associated cytokines are modulated in the colonic tissue of $I B D$ mice. Previous studies have demonstrated a decrease in Treg cell count and an increase in Th17 (29). Therefore, the present study investigated the mRNA expression of signature transcription factors of Th17 and Treg cells, and associated cytokines, in the colonic tissue. RT-qPCR analysis demonstrated that the mRNA expression of IL-17, retinoic acid related orphan receptor- $\gamma \mathrm{t}$ (ROR- $\gamma \mathrm{t}), \mathrm{TNF}-\alpha$, Foxp3, and FGL2 in the TNBS group were markedly increased compared with the control group (Fig. 5).

Levels of FGL2 are increased in plasma from TNBS-induced mice in addition to elevated pro-inflammatory cytokine expression. In order to determine whether the expression of FGL2 and IL-17 was altered in the peripheral blood of TNBS-induced mice, the plasma expression of FGL2, IL-17 and TNF- $\alpha$ was analyzed. As presented in Fig. 6, significantly increased FGL2 secretion and increased production of IL-17 and TNF- $\alpha$ in plasma, were detected in the TNBS group compared with the control group.

Distinct frequencies of splenic Treg/Th17 cells in TNBS-induced mice. It has been hypothesized that an imbalance of Treg and Th17 cells may be associated with the 
A

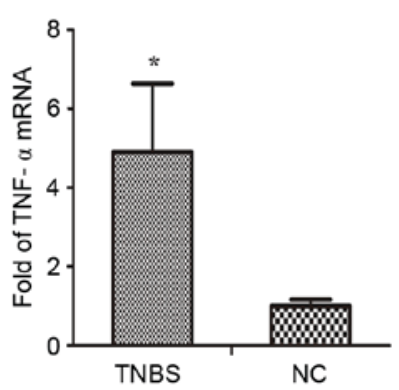

D

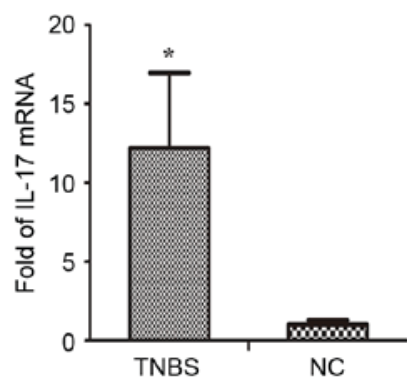

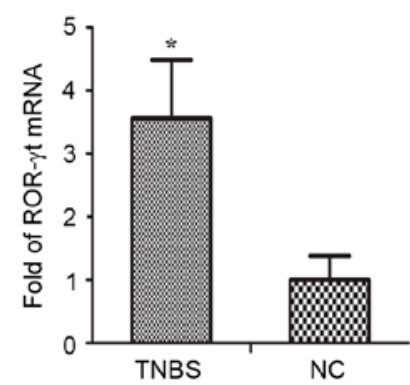

E
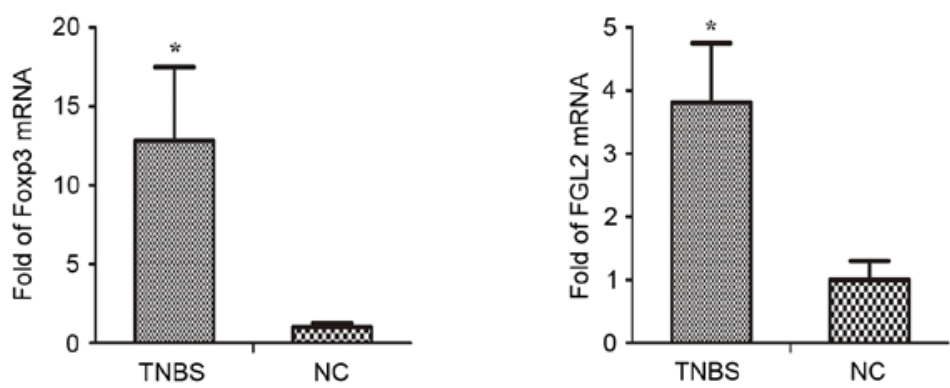

Figure 5. Altered gene expression in the colon of TNBS-induced inflammatory bowel disease mice. Reverse transcription-quantitative polymerase chain reaction analysis was performed of the mRNA expression of (A) TNF- $\alpha$, (B) IL-17, (C) ROR- $\gamma$ t, (D) Foxp3 and (E) FGL2 in colonic tissue. "P<0.05 vs. NC. $\mathrm{n}=8$ mice/group. TNBS, trinitro-benzene-sulfonic acid; NC, normal control; TNF- $\alpha$, tumor necrosis factor- $\alpha$; FGL2, fibrinogen-like protein 2; IL-17, interleukin-17; ROR- $\gamma \mathrm{t}$, retinoic acid related orphan receptor- $\gamma \mathrm{t}$; Foxp3, forkhead box protein 3.

pathogenesis of IBD $(29,30)$. The aim of the present study was to further investigate the polarization of $\mathrm{CD} 4^{+} \mathrm{T}$ cells in spleens from TNBS-induced mice (Fig. 7). Flow cytometric analysis demonstrated that $\mathrm{CD} 4{ }^{+} \mathrm{CD} 25^{+} \mathrm{Foxp}^{+}$(Treg) cells were markedly decreased, while CD4+IL-17 ${ }^{+}$(Th17) cells were significantly increased following TNBS challenge (Fig. 7E). In parallel, the expression of Th17- and Treg-specific transcription factors and associated cytokines in the splenic lymphocytes were analyzed using RT-qPCR. The results of the present study indicated that the mRNA expression of IL-17 and ROR- $\gamma t$ in the TNBS group was markedly increased compared with the control group, while Foxp3 and FGL2 mRNA were significantly downregulated (Fig. 7A-D).

\section{Discussion}

The etiopathogenesis of IBD is complex, involving defects in the mucosal barrier and immune system. In the digestive system, in the context of IBD, destruction of immune tolerance results in pathological inflammation. An excessive inflammatory milieu, and insufficient functioning or quantity of cellular constituents that downregulate the immune response, may be the principal causes for the imbalance of the intestinal immune system. Previous studies have demonstrated that a lack of equilibrium between Treg and Th17 cells serves a role in inflammation in $\operatorname{IBD}(31,32)$.

Th17 cells and associated pro-inflammatory mediators, including IL-17, IL-12, IL-23 and IL-26, have been identified to be novel factors implicated in the pathogenesis of autoimmune disease. As the receptors for the above cytokines are expressed by intestinal epithelial cells, Th17 cells exert an important impact on the development and progression of IBD. Among several cytokines, IL-17 response is thought to act as the initial trigger of colitis (11,30,33-35). While Th17 cells facilitate intestinal inflammation in IBD, Treg cells exhibit effective anti-inflammatory properties by inhibiting the cell proliferation and cytokine secretion of Th1 and Th2 cells $(30,32,36)$. Previous studies have indicated that Treg cells serve an important role in the maintenance of mucosal tolerance and are able to cure experimentally-induced colitis. In addition, the transcription factor of functional Treg cells, Foxp3, is associated with their regulatory effect $(32,37,38)$. Certain studies have demonstrated that important mediators of Treg cells, including transforming growth factor- $\beta$ (TGF- $\beta$ ), IL-35 and IL-10, serve an important role in the anti-inflammatory effect $(39,40)$; however, a number of studies have demonstrated that inhibitors of these molecules are unable to block the inhibitory effect of Treg cells $(39,40)$. It has been previously demonstrated that FGL2, secreted by T cells, is involved in the immunomodulatory activity of Treg cells $(39,41)$. One hypothesis is that FGL2 indirectly inhibits $\mathrm{T}$ cell proliferation and immune activity by suppressing the nuclear factor $-\kappa \mathrm{B}$ signaling pathway, to inhibit the maturity of dendritic cells. FGL2 directly decreases B cell immune responses through the induction of B cell apoptosis (42). Th17 are able to regulate the development of Treg cells, and vice versa, to maintain immune homeostasis and accomplish pathogen clearance (43). TGF- $\beta$ has been proposed to be the essential common factor for the proliferation of Th17 and Treg cells, and not only an anti-inflammatory cytokine produced by Treg cells. It has been reported that TGF- $\beta$ promotes the differentiation of Treg cells by inducing Foxp3 expression, whereas it promotes the differentiation of Th17 cells with concurrent administration of IL-6 and TGF- $\beta$ (43). 

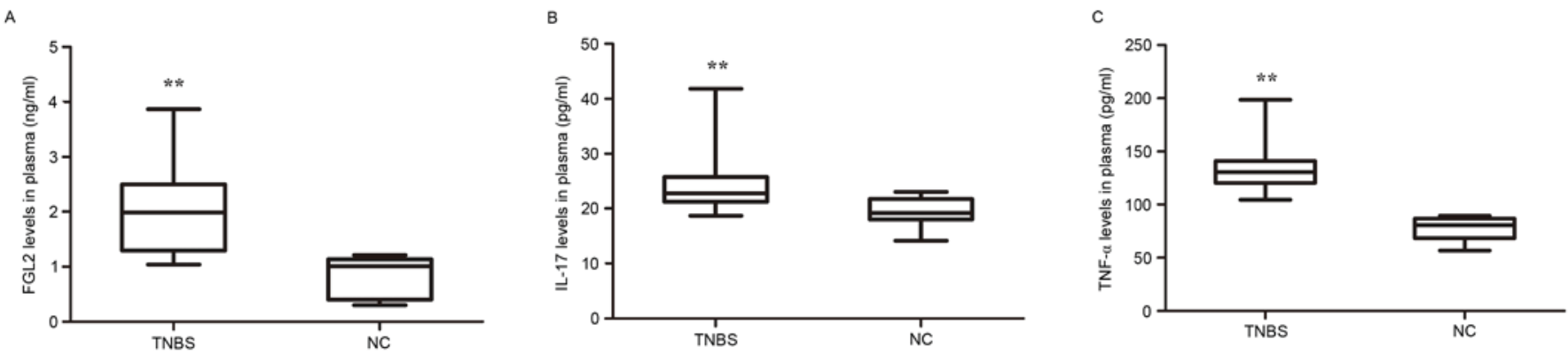

Figure 6. ELISA analysis of plasma from TNBS-induced inflammatory bowel disease mice. ELISA analysis of (A) FGL2, (B) IL-17 and (C) TNF- $\alpha$ in plasma from each group is presented. ${ }^{* *} \mathrm{P}<0.001$ vs. NC. $\mathrm{n}=8$ mice/group. TNBS, trinitro-benzene-sulfonic acid; NC, normal control; TNF- $\alpha$, tumor necrosis factor- $\alpha$; FGL2, fibrinogen-like protein 2; IL-17, interleukin-17.

A
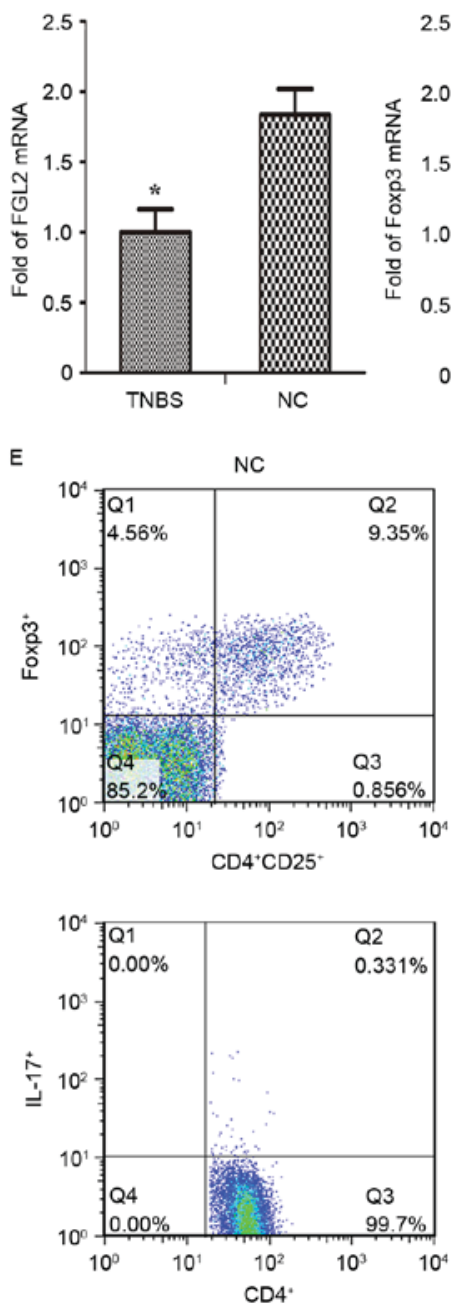

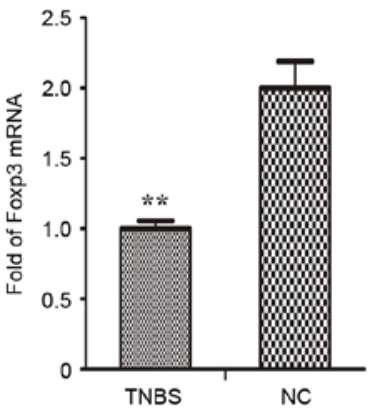

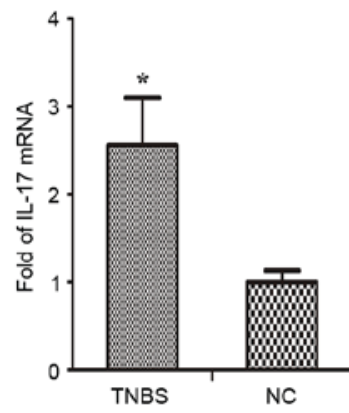

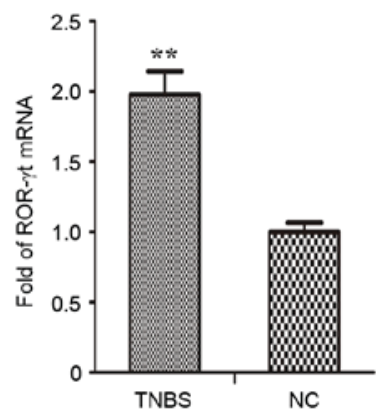

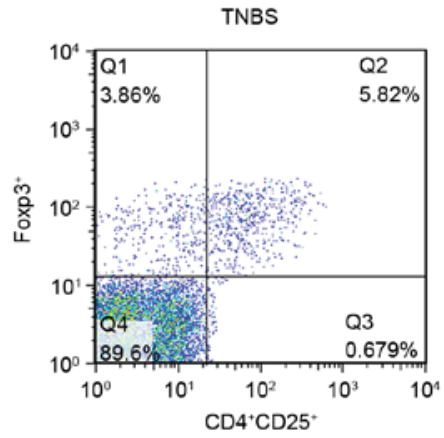
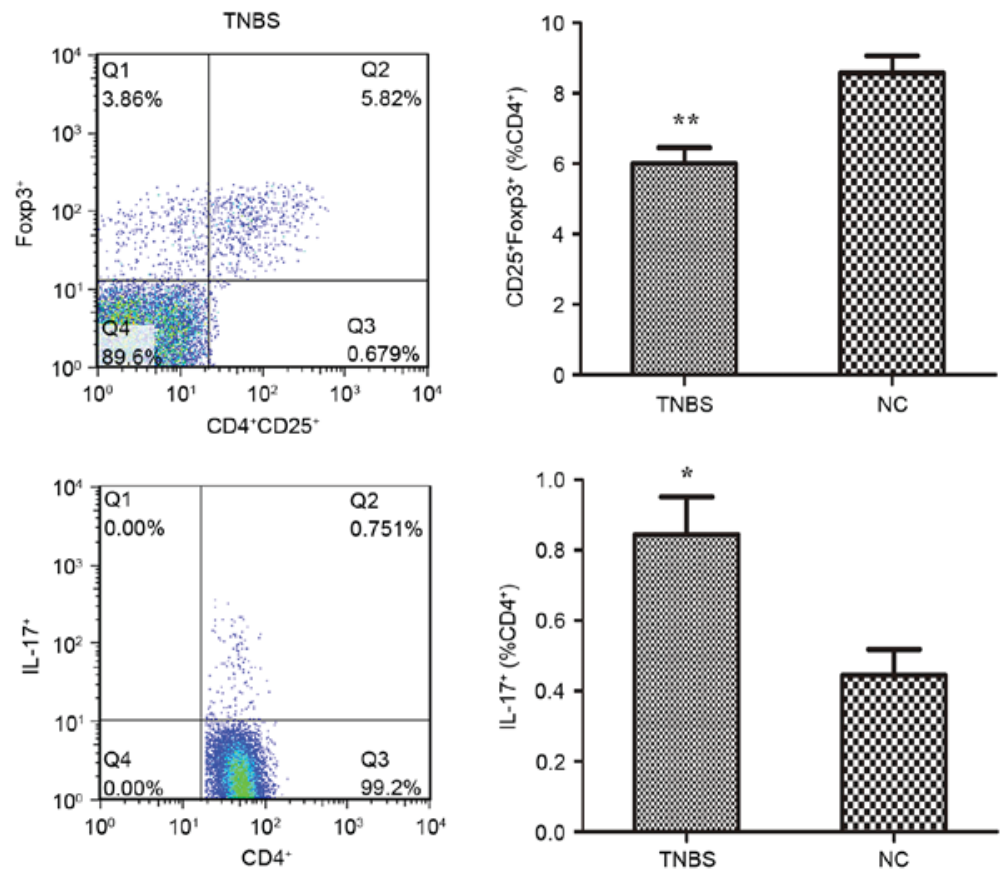

Figure 7. Analysis of gene expression in splenic lymphocytes of TNBS-induced inflammatory bowel disease mice. Reverse transcription-quantitative polymerase chain reaction analysis was performed to assess the mRNA expression of (A) FGL2, (B) Foxp3, (C) IL-17 and (D) ROR- $\gamma$ t in splenic lymphocytes. (E) Flow cytometric analysis of regulatory $\mathrm{T}$ and $\mathrm{T}$ helper 17 cells was performed and quantified. ${ }^{*} \mathrm{P}<0.05,{ }^{* *} \mathrm{P}<0.001 \mathrm{vs}$. NC. $\mathrm{n}=8 \mathrm{mice} / \mathrm{group}$. TNBS, trinitro-benzene-sulfonic acid; NC, normal control; TNF- $\alpha$, tumor necrosis factor- $\alpha$; FGL2, fibrinogen-like protein 2; IL-17, interleukin-17; ROR- $\gamma$ t, retinoic acid related orphan receptor- $\gamma \mathrm{t}$; Foxp3, forkhead box protein 3.

In a previous study, the expression of FGL2 was detected in peripheral blood samples and intestinal tissue from patients with IBD, and the results demonstrated that the level of FGL2 was markedly increased in the active period of IBD and positively correlated to the disease activity index (22). Based on the above previous results, it may be hypothesized that the immunological activity of FGL2 may contribute to the pathogenesis of IBD by maintaining the balance between Treg and Th17 cells. In the present study, the expression of FGL2 and other cytokines, which are secreted by Treg and Th17 cells, was measured in the TNBS-induced colitis model. The clinical manifestation, macroscopic scoring and histological examination in the 
animal model indicated that the TNBS-induced experimental animals conformed to the features of IBD. The experimental group was observed to exhibit an increased expression of TNF- $\alpha$, which serves an explicit pathogenic role in IBD. The elevated expression of FGL2 and IL-17 was observed in the colon tissue of IBD mice compared with the control group. Similar to the lesion tissue in the colon, an increase in FGL2 and IL-17 was additionally observed in the serum of IBD mice. In addition, the proportion of Treg and Th17 cells was detected in the IBD mice and control group. The results of the present study exhibited an elevated frequency of Th17 cells, in addition to a decreased frequency of Treg cells. This result is consistent with other previous studies $(29,33,44)$. The results of the present study demonstrated that the mRNA level of ROR- $\gamma \mathrm{t}$, the transcription factor which guides Th17 differentiation, was increased in the colon tissue and splenic lymphocytes of IBD mice. However, Foxp3 mRNA was minimally expressed in splenic lymphocytes, although it was highly expressed in the colon tissue, suggesting that Treg cells may be recruited to the intestinal lesion area in order to repress the pro-inflammatory response (22). Consistent with Foxp3, increased expression of FGL2 mRNA was demonstrated in the colon of IBD mice; however, levels in the splenic lymphocytes were decreased. The alteration in levels of FGL2 was hypothesized to reflect their accumulation in the inflamed region of the intestinal tract (22). Therefore, upregulation of FGL2 in the peripheral blood and intestinal lesions of IBD mice may counter the pro-inflammatory role of the effecter $\mathrm{T}$ cells, so as to regain balance in the intestinal immune system. However, the increased level of FGL2 appears to be an insufficient counter regulation, which fails to inhibit the progression of IBD.

In conclusion, it was observed that the intestinal and peripheral expression of FGL2 and IL-17 in TNBS-induced colitis mice was significantly increased compared with healthy mice, and that the balance between Th17 and Treg cells was disrupted in the spleen of the model animals. The present study demonstrated that FGL2 was associated with the immunopathogenesis of IBD and with Th17/Treg balance. The present results suggested that the increased level of Treg-expressed FGL2 may be a potential biomarker for the assessment of IBD and may lead to a novel therapeutic strategy, although this hypothesis requires further investigation. Therefore, future studies will aim to gain an insight into the feasible mechanisms underlying the immunosuppressive effect of FGL2 in IBD.

\section{Acknowledgements}

The authors of the present study would like to acknowledge Professor Huiping Zhou of the Department of Microbiology and Immunology, Virginia Commonwealth University (Richmond, VA, USA) for assistance with professional English-language editing. The present study was supported by the Public Technology Research Project of Zhejiang Province (grant no. 2015C33120) and the Natural Science Foundation of China (grant no. 81570495).

\section{References}

1. Geremia A, Biancheri P, Allan P, Corazza GR and Di Sabatino A: Innate and adaptive immunity in inflammatory bowel disease. Autoimmun Rev 13: 3-10, 2014.
2. Dothel G, Vasina V, Barbara G and De Ponti F: Animal models of chemically induced intestinal inflammation: Predictivity and ethical issues. Pharmacol Ther 139: 71-86, 2013.

3. Molodecky NA, Soon IS, Rabi DM, Ghali WA, Ferris M, Chernoff G, Benchimol EI, Panaccione R, Ghosh S, Barkema HW and Kaplan GG: Increasing incidence and prevalence of the inflammatory bowel diseases with time, based on systematic review. Gastroenterology 142: 46-54.e42; quiz e30, 2012.

4. Prideaux L, Kamm MA, De Cruz PP, Chan FK and Ng SC: Inflammatory bowel disease in Asia: A systematic review. J Gastroenterol Hepatol 27: 1266-1280, 2012.

5. Zou Y, Li WY, Wan Z, Zhao B, He ZW, Wu ZG, Huang GL, Wang J, Li BB, Lu YJ, et al: Huangqin-tang ameliorates TNBS-induced colitis by regulating effector and regulatory CD4 (+) T cells. Biomed Res Int 2015: 102021, 2015.

6. Strober W, Fuss I and Mannon P: The fundamental basis of inflammatory bowel disease. J Clin Invest 117: 514-521, 2007

7. Matricon J, Barnich $\mathrm{N}$ and Ardid D: Immunopathogenesis of inflammatory bowel disease. Self Nonself 1: 299-309, 2010.

8. Maynard CL and Weaver CT: Intestinal effector T cells in health and disease. Immunity 31: 389-400, 2009.

9. Zhu J, Yamane H and Paul WE: Differentiation of effector CD4 T cell populations $\left(^{*}\right)$. Annu Rev Immunol 28: 445-489, 2010.

10. Almeida Cde S, Andrade-Oliveira V, Câmara NO, Jacysyn JF and Faquim-Mauro EL: Crotoxin from crotalus durissus terrificus is able to down-modulate the acute intestinal inflammation in mice. PLoS One 10: e0121427, 2015.

11. Dambacher J, Beigel F, Zitzmann K, De Toni EN, Göke B, Diepolder HM, Auernhammer CJ and Brand S: The role of the novel Th17 cytokine IL-26 in intestinal inflammation. Gut 58: 1207-1217, 2009.

12. Geremia A and Jewell DP: The IL-23/IL-17 pathway in inflammatory bowel disease. Expert Rev Gastroenterol Hepatol 6: 223-237, 2012.

13. Valencia X, Stephens G, Goldbach-Mansky R, Wilson M, Shevach EM and Lipsky PE: TNF downmodulates the function of human CD4+CD25hi T-regulatory cells. Blood 108: 253-261, 2006.

14. Khor B, Gardet A and Xavier RJ: Genetics and pathogenesis of inflammatory bowel disease. Nature 474: 307-317, 2011.

15. Cătană CS, Berindan Neagoe I, Cozma V, Magdas C, Tăbăran F and Dumitraşcu DL: Contribution of the IL-17/IL-23 axis to the pathogenesis of inflammatory bowel disease. World J Gastroenterol 21: 5823-5830, 2015.

16. Himmel ME, Hardenberg G, Piccirillo CA, Steiner TS and Levings MK: The role of T-regulatory cells and Toll-like receptors in the pathogenesis of human inflammatory bowel disease. Immunology 128: 145-153, 2008.

17. Chruscinski A, Sadozai H, Rojas-Luengas V, Bartczak A, Khattar R, Selzner N and Levy GA: Role of regulatory T cells (Treg) and the treg effector molecule fibrinogen-like protein 2 in alloimmunity and autoimmunity. Rambam Maimonides Med J 6, 2015.

18. Bilate AM and Lafaille JJ: Induced CD4+Foxp3+ regulatory $\mathrm{T}$ cells in immune tolerance. Annu Rev Immunol 30: 733-758, 2012.

19. Joller N, Lozano E, Burkett PR, Patel B, Xiao S, Zhu C, Xia J, Tan TG, Sefik E, Yajnik V, et al: Treg cells expressing the coinhibitory molecule TIGIT selectively inhibit proinflammatory Th1 and Th17 cell responses. Immunity 40: 569-581, 2014.

20. Shalev I, Liu H, Koscik C, Bartczak A, Javadi M, Wong KM, Maknojia A, He W, Liu MF, Diao J, et al: Targeted deletion of fg12 leads to impaired regulatory T cell activity and development of autoimmune glomerulonephritis. J Immunol 180: 249-260, 2008.

21. Chan CW, Kay LS, Khadaroo RG, Chan MW, Lakatoo S, Young KJ, Zhang L, Gorczynski RM, Cattral M, Rotstein O and Levy GA: Soluble fibrinogen-like protein 2/fibroleukin exhibits immunosuppressive properties: Suppressing T cell proliferation and inhibiting maturation of bone marrow-derived dendritic cells. J Immunol 170: 4036-4044, 2003.

22. Dong X, Ye X, Chen X, Chen T, Xie S, Li Q, Lin X and Huang Z: Intestinal and peripheral fibrinogen-like protein 2 expression in inflammatory bowel disease. Dig Dis Sci 59: 769-777, 2014.

23. Alex P, Zachos NC, Nguyen T, Gonzales L, Chen TE, Conklin LS, Centola M and Li X: Distinct cytokine patterns identified from multiplex profiles of murine DSS and TNBS-induced colitis. Inflamm Bowel Dis 15: 341-352, 2009. 
24. Wirtz S, Neufert C, Weigmann B and Neurath MF: Chemically induced mouse models of intestinal inflammation. Nat Protoc 7: 541-546, 2007.

25. Glauben R, Batra A, Stroh T, Erben U, Fedke I, Lehr HA, Leoni F, Mascagni P, Dinarello CA, Zeitz M and Siegmund B: Histone deacetylases: Novel targets for prevention of colitis-associated cancer in mice. Gut 57: 613-622, 2008.

26. Bell CJ, Gall DG and Wallace JL: Disruption of colonic electrolyte transport in experimental colitis. Am J Physiol 268 G622-G630, 1995.

27. Scheiffele F and Fuss IJ: Induction of TNBS colitis in mice. Curr Protoc Immunol: Chapter 15, Unit 15. 19, 2002.

28. Livak KJ and Schmittgen TD: Analysis of relative gene expression data using real-time quantitative PCR and the 2(-Delta Delta C(T)) method. Methods 25: 402-408, 2001.

29. Eastaff-Leung N, Mabarrack N, Barbour A, Cummins A and Barry S: Foxp3+ regulatory T cells, Th17 effector cells, and cytokine environment in inflammatory bowel disease. J Clin Immunol 30: 80-89, 2010

30. Chaudhry A, Rudra D, Treuting P, Samstein RM, Liang Y, Kas A and Rudensky AY: CD4+ regulatory T cells control TH17 responses in a Stat3-dependent manner. Science 326: 986-991, 2009.

31. Kaser A, Zeissig S and Blumberg RS: Inflammatory bowel disease. Annu Rev Immunol 28: 573-621, 2010.

32. Veltkamp C, Anstaett M, Wahl K, Möller S, Gangl S, Bachmann O, Hardtke-Wolenski M, Länger F, Stremmel W, Manns MP, et al: Apoptosis of regulatory $\mathrm{T}$ lymphocytes is increased in chronic inflammatory bowel disease and reversed by anti-TNF $\alpha$ treatment. Gut 60: 1345-1353, 2011.

33. Brand S: Crohn's disease: Th1, Th17 or both? the change of a paradigm: New immunological and genetic insights implicate Th17 cells in the pathogenesis of Crohn's disease. Gut 58: 1152-1167, 2009.

34. Kugathasan S, Saubermann LJ, Smith L, Kou D, Itoh J, Binion DG, Levine AD, Blumberg RS and Fiocchi C: Mucosal T-cell immunoregulation varies in early and late inflammatory bowel disease. Gut 56: 1696-1705, 2007.
35. Wilson NJ,Boniface K, Chan JR,McKenzie BS,Blumenschein WM, Mattson JD, Basham B, Smith K, Chen T, Morel F, et al: Development, cytokine profile and function of human interleukin 17-producing helper T cells. Nat Immunol 8: 950-957, 2007.

36. Vignali DA, Collison LW and Workman CJ: How regulatory T cells work. Nat Rev Immunol 8: 523-532, 2008.

37. Maul J, Loddenkemper C, Mundt P, Berg E, Giese T, Stallmach A, Zeitz $M$ and Duchmann R: Peripheral and intestinal regulatory $\mathrm{CD} 4+\mathrm{CD} 25$ high $\mathrm{T}$ cells in inflammatory bowel disease. Gastroenterology 128: 1868-1878, 2005.

38. Hori S, Nomura T and Sakaguchi S: Control of regulatory $\mathrm{T}$ cell development by the transcription factor Foxp3. Science 299: 1057-1061, 2003

39. Shalev I, Wong KM, Foerster K, Zhu Y, Chan C, Maknojia A, Zhang J, Ma XZ, Yang XC, Gao JF, et al: The novel CD4+CD25+ regulatory $\mathrm{T}$ cell effector molecule fibrinogen-like protein 2 contributes to the outcome of murine fulminant viral hepatitis. Hepatology 49: 387-397, 2009.

40. Miyara M and Sakaguchi S: Natural regulatory $\mathrm{T}$ cells: Mechanisms of suppression. Trends Mol Med 13: 108-116, 2007.

41. Foerster K, Helmy A, Zhu Y, Khattar R, Adeyi OA, Wong KM, Shalev I, Clark DA, Wong PY, Heathcote EJ, et al: The novel immunoregulatory molecule FGL2: A potential biomarker for severity of chronic hepatitis C virus infection. J Hepatol 53 608-615, 2010.

42. Liu H, Shalev I, Manuel J, He W, Leung E, Crookshank J, Liu MF, Diao J, Cattral M, Clark DA, et al: The FGL2-FcgammaRIIB pathway: A novel mechanism leading to immunosuppression. Eur J Immunol 38: 3114-3126, 2008.

43. Bettelli E, Carrier Y, Gao W, Korn T, Strom TB, Oukka M, Weiner HL and Kuchroo VK: Reciprocal developmental pathways for the generation of pathogenic effector TH17 and regulatory T cells. Nature 441: 235-238, 2006.

44. Elshal MF, Aldahlawi AM, Saadah OI and McCoy JP: Reduced dendritic cells expressing CD200R1 in children with inflammatory bowel disease: Correlation with Th17 and regulatory T cells. Int J Mol Sci 16: 28998-29010, 2015. 